\title{
Deficient Antigen-presenting Cell Function in Multiple Genetic Complementation Groups of Type II Bare Lymphocyte Syndrome
}

\author{
Susan Kovats, * Gerald T. Nepom, ${ }^{*}$ Marilyn Coleman, Barbara Nepom, ${ }^{\star}$ William W. Kwok, and Janice S. Blum ${ }^{*}$ \\ Immunology and Diabetes Programs, Virginia Mason Research Center, Seattle, Washington 98101; and Departments of *Immunology \\ and ${ }^{\ddagger}$ Pediatrics, University of Washington School of Medicine, Seattle, Washington 98195
}

\begin{abstract}
The absence of HLA class II gene expression in type II bare lymphocyte syndrome (BLS) results from defective transcriptional activation of class II histocompatibility genes. Genetic studies have revealed that distinct defects in multiple trans-acting factors result in the immunodeficient BLS phenotype. We studied antigen-presenting cell (APC) function in DR-transfected BLS cells derived from multiple complementation groups. Each BLS cell line displayed the same defective APC phenotype: an inability to mediate class II-restricted presentation of exogenous protein antigens, and structurally altered class II $\alpha \beta$ dimers. Expression of the HLA class II-like genes DMA and DMB, previously implicated in antigen presentation, was reduced or absent in the BLS cells. Fusion of BLS cells with cell line 721.174, which has a genomic deletion of HLA class II genes, coordinately restores class II structural gene and DM gene expression and a wild-type APC phenotype. Thus each of the molecular defects that silences class II structural gene transcription also results in a defective $\mathrm{APC}$ phenotype, providing strong evidence for coregulation of these two functionally linked pathways. (J. Clin. Invest. 1995. 96:217-223.) Key words: gene expression regulation • genes MHC class II • immunocompetence $\cdot$ disease $\cdot$ HLA-DR antigen
\end{abstract}

\section{Introduction}

Type II bare lymphocyte syndrome (BLS), ${ }^{1}$ or HLA class IIdeficient combined immunodeficiency, is an autosomal recessive disorder in which the HLA class II structural genes are not transcribed in any tissue either constitutively or in response to inducing stimuli $(1,2)$. The resulting absence of class II histocompatibility antigen expression on antigen-presenting cells, such as B lymphocytes and cells of the macrophage lin-

Address correspondence to Susan Kovats, Virginia Mason Research Center, 1000 Seneca St., Seattle, WA 98101. Phone: 206-223-8813; FAX: 206-223-7543. J. S. Blum's current address is Dept. of Microbiology and Immunology, Indiana University School of Medicine, Indianapolis, IN 46202.

Received for publication 26 January 1995 and accepted in revised form 24 March 1995.

1. Abbreviations used in this paper: APC, antigen-presenting cells; BLCL, B lymphoblastoid cell line; BLS, bare lymphocyte syndrome; CLIP, invariant chain-derived peptide; HA, influenza hemaglutinin; TT, tetanus toxoid.

J. Clin. Invest.

(c) The American Society for Clinical Investigation, Inc.

0021-9738/95/07/0217/07 \$2.00

Volume 96 , July $1995,217-223$ eage, significantly impairs cellular and humoral immune responses (reviewed in reference 3 ). Thus, BLS immunodeficiency is characterized by a paucity of $\mathrm{T}$ lymphocyte responses and impaired antibody production upon challenge with foreign antigens, leading to extreme susceptibility to viral, bacterial, and fungal infections. These pathophysiologic defects in BLS have been directly attributed to a lack of HLA class II-restricted antigen presentation to $\mathrm{T}$ lymphocytes.

The absence of HLA class II gene expression results from mutations in transactivator genes necessary to mediate transcription of the coordinately regulated class II genes $(2,4,5)$. Genetic studies have revealed that at least four distinct molecular defects manifest as the class II null phenotype of BLS patient cells $(6,7)$. We have previously demonstrated that a BLS cell line (BLS-1) isolated from a patient from one of these complementation groups, following transfection with wild-type class II genes, has defects in class II-mediated presentation of native exogenous antigens and structurally altered class II $\alpha \beta$ molecules (8). This finding suggested that the molecular pathways regulating HLA class II structural gene expression and those modulating pathways of class II functional antigen processing and presentation share common elements, possibly coregulated by the transcriptionally active elements missing in patients with BLS.

Presentation of exogenous foreign antigens by antigen-presenting cells (APC) is a complex process, in which antigens are proteolytically processed to peptide epitopes that are then bound by class II molecules and displayed at the cell surface for recognition by antigen-specific $\mathrm{CD} 4^{+}$helper $\mathrm{T}$ lymphocytes (reviewed in reference 9). Mutational analyses have shown that genes essential for appropriate class II-mediated antigen presentation, recently identified as HLA-DMA and -DMB $(10,11)$, map within the class II region of the MHC (12-15), raising the possibility that this clustering of genes encoding both class II structural proteins and accessory molecules required for their antigen-presenting function would ensure their coordinate regulation. In addition, there may be multiple genes outside the MHC that are required for optimal antigen presentation, such as the invariant chain (16), that share regulatory elements with HLA class II genes.

To analyze the coregulation of these phenotypes, class II transcription and antigen presentation, we transfected class II HLA-DR molecules into different BLS cell lines, thereby restoring structural gene expression. Several structural and functional aspects of class II-dependent antigen processing and presentation were then tested in these cells. DR-transfected B cell lines, BLS-1, SJO, and 6.1.6, representative of three distinct BLS complementation groups, showed the same defective APC phenotype: $(a)$ inability to participate in the class II-mediated presentation of exogenous native protein antigens although presentation of preprocessed peptides was efficient; and $(b)$ structurally abnormal class II $\alpha \beta$ dimers with reduced stability in SDS. The distinct transactivators defective in each of the different 
BLS groups were required for expression of genes involved in class II-mediated antigen presentation, indicating a common transcriptional pathway coordinate with structural class II gene expression.

\section{Methods}

Cell lines. BLS-1 and BLS-2 (17) (kindly provided by J. Lee, Memorial Sloan Kettering Cancer Center, New York), and SJO (18) are HLA class II-null B-LCL generated from cells of patients with type II Bare Lymphocyte Syndrome and are members of complementation groups I, II, and IV, respectively (7). The class II-null B-LCL 6.1.6 was generated after in vitro mutagenesis (19). 6.1.6 (classified as complementation group III [7]) has a mutation resulting in the absence of class II transcription and thus exhibits the class II null phenotype of BLS patient cells. T2 $(.174 \times$ CEM.T2 $)$ is a human cell hybrid that lacks all four copies of the MHC class II region on chromosome 6 (20). Class II DR $\alpha$ (DRA1) and DR $\beta$ (DRB 1 *0401) cDNA encoding DR4w4 molecules were introduced into SJO and 6.1.6 using retrovirus-mediated gene transfer (21). The BLS-1.DR4w4, BLS-1.DR3, BLS-1.DR5, and T2.DR4w4 transferent cells have been described (8). BLS-1.DR4w14 transferent cells were generated by introduction of DRA1 and DRB $1 * 0404$ genes. 721.174 is a mutant (generated in vitro) human B cell line that has a homozygous deletion of the MHC class II region (22); .174 cells containing either neomycin-resistance or gpt (guanine phosphoribosyltransferase) genes were kindly provided by R. Greenwood and R. DeMars (University of Wisconsin, Madison, WI). The DR4w4 B cell lines Priess and Savc, and DR3 B cell lines Mat and Vavy were used as wild-type controls. Cell lines were grown in RPMI1640 medium plus 10\% FCS (Hyclone, Logan, UT).

Antibody binding assays. mAb binding to live cells was detected by flow cytometry using a FACScan (Becton Dickinson and Co., Cockeysville, MD). The anti-DR mAb L243 (23) and anti-DR3 mAb 16.23 (24) were used at saturating concentrations and detected using FITCconjugated goat anti-mouse Ig (Jackson ImmunoResearch Labs. Inc. West Grove, PA).

Assays with $\mathrm{T}$ cell hybridomas. Antigen-specific $\mathrm{T}$ cell hybridomas were generously supplied by P. Whiteley (Merck Research Labs., Rahway, NJ). The DR4w4-tetanus-specific T cell hybridoma 49.23.2 was generated after tetanus immunization of a DR4w4 transgenic mouse (25). Formalin-fixed tetanus toxoid (TT) (Wyeth Laboratories, Philadelphia, PA) was digested with $N$-tosyl-L-phenylalanine chloromethyl ketone trypsin. The presence of antigenic peptides in the tryptic digest of TT was confirmed by incubating the digested preparation with paraformaldehyde-fixed APCs. APC $\left(10^{5}\right)$, TT antigen $(0.4 \mu \mathrm{M}$ of native or trypsin-digested), and 49.23.2 T cells $\left(10^{5}\right)$ were incubated together for $24 \mathrm{~h}$. In some experiments, APC were preincubated with either 0.4 or $0.8 \mu \mathrm{M}$ of TT antigen (native or trypsin digested) for $3 \mathrm{~h}$ at $37^{\circ} \mathrm{C}$ and subsequently washed twice with medium before plating in wells with $\mathrm{T}$ cells.

The DR4w4-hemagglutinin-specific $T$ cell hybridoma 50.84 .17 was generated after immunization of a DR4w4 transgenic mouse with fixed influenza virus; the $\mathrm{T}$ cell hybridoma is specific for the influenza hemagglutinin peptide epitope HA 307-319 (PKYVKQNTLKLAT). APC $\left(10^{5}\right)$ were incubated with either $1.0 \mu \mathrm{M}$ HA 307-319 peptide or 50 $\mu \mathrm{g} / \mathrm{ml}$ fixed A/Mississippi/1/85 (H3N2) influenza virus (Connaught Laboratories, Swiftwater, PA) for $3 \mathrm{~h}$ at $37^{\circ} \mathrm{C}$ and subsequently washed twice with medium before plating in wells with $50.84 .17 \mathrm{~T}$ cells $\left(10^{5}\right)$. $\mathrm{T}$ cell $\mathrm{IL}-2$ and $\mathrm{IL}-4$ production was determined by survival of an IL-2/IL-4-dependent cell line, HT-2, measured using $\left[{ }^{3} \mathrm{H}\right]$ thymidine incorporation $(0.5 \mu \mathrm{Ci} /$ well $)(26)$. The $\mathrm{T}$ cell hybridomas did not produce lymphokines in response to APCs in the absence of antigen. Assays were repeated three to four times with similar results.

$m R N A$ analyses. RNA was isolated from 5-10 $\times 10^{6}$ cells, and cDNA was made from $1.0 \mu \mathrm{g}$ of RNA using reverse transcriptase. Primer-directed amplification of cDNA was accomplished using PCR. $5 '$ and 3 ' primers for amplification of DMA cDNA were: 5'-ACTTTTCCCAGAACACTCGG and 5'-CTGGAAGCTGAGTCCATCG and the expected product is $340 \mathrm{bp} .5^{\prime}$ and $3^{\prime}$ primers for amplification of DMB cDNA were: 5'-ACAGCACCTCAACCAAAAAGA and 5'-GGGGTTAAGGCTAAATGGGA and the expected product is $320 \mathrm{bp}$. DMA and DMB cDNA were amplified for 30 cycles (denaturation for $1 \mathrm{~min}$ at $93^{\circ} \mathrm{C}$, annealing for $1 \mathrm{~min}$ at $55^{\circ} \mathrm{C}$ and polymerization for $1 \mathrm{~min}$ at $72^{\circ} \mathrm{C}$ ). The reaction products were visualized by agarose gel electrophoresis and staining with ethidium bromide. PCR products were sized using a 1-kb DNA ladder (GIBCO BRL, Gaithersburg, MD). The identity of the putative DMA band amplified in BLS-1 was confirmed by DNA sequence analysis. The presence of intact cDNA in samples derived from cellular RNA was confirmed by the presence of a product amplified using primers specific for the actin gene. A higher molecular weight band amplified in some of the RNA samples is likely to be resulting from genomic DNA contamination of the RNA sample, since the size of the product $(\sim 1 \mathrm{~kb}$ for DMA and $\sim 1.5 \mathrm{~kb}$ for DMB $)$ corresponds to the size of the product directly amplified from genomic DNA of Vavy.

Cell fusions. Fusions between BLS-1 (DR3,5) and .174 or between SJO (DR5,7) and .174 were accomplished using 50\% polyethylene glycol-1500, 5\% DMSO (4). Before cell hybridization, genes encoding drug resistance were introduced into the cell lines as follows: BLS-1, hygromycin-resistance gene; .174, neomycin-resistance or gpt genes; and SJO, neomycin-resistance gene linked to a DRB $1 * 0401$ gene. The BLS-1 $\times .174$ (neo) fused cells were selected using hygromycin B (Calbiochem Novabiochem, La Jolla, CA) $(150 \mu \mathrm{g} / \mathrm{ml})$ and G418 $($ GIBCO BRL) $(1 \mathrm{mg} / \mathrm{ml})$. The SJO $\times .174(g p t)$ fused cells were selected for using G418 and mycophenolic acid ( $10 \mu \mathrm{g} / \mathrm{ml})$ plus xanthine $(6 \mu \mathrm{g} / \mathrm{ml})$ (Sigma Immunochemicals, St. Louis, MO). Stable independently fused drug-resistant populations were analyzed. The BLS$1 \times$ SJO hybrids have been described (8).

Analyses of HLA class II dimers using immunoblotting. Cells were lysed at $5 \times 10^{7} / \mathrm{ml}$ in $1 \% \mathrm{NP}-40,0.15 \mathrm{M} \mathrm{NaCl}, 5 \mathrm{mM}$ EDTA, $50 \mathrm{mM}$ Tris-Cl, pH 7.2, $1.0 \mathrm{mM}$ PMSF, $1.0 \mu \mathrm{g} / \mathrm{ml}$ leupeptin, and $1.0 \mu \mathrm{g} / \mathrm{ml}$ pepstatin for $30 \mathrm{~min}$ on ice; protease inhibitors were obtained from Boehringer Mannheim Corp. (Indianapolis, IN). To detect HLA-DR $\alpha \beta$ dimers, $50 \mu \mathrm{g}$ of total cell lysate was mixed with the appropriate volume of $4 \times$ nonreducing SDS sample buffer $(1 \times$ concentration: 62.5 $\mathrm{mM}$ Tris-Cl, $\mathrm{pH} 6.8,0.2 \%$ SDS, $10 \%$ glycerol) and incubated for 30 min at room temperature. To detect HLA-DR $\beta$ monomers, $25 \mu \mathrm{g}$ of total cell lysate was mixed with an appropriate volume of $4 \times$ reducing SDS sample buffer $(1 \times$ concentration as above plus $100 \mathrm{mM}$ DTT $)$ and boiled for $10 \mathrm{~min}$. Samples were electrophoresed on a 10\% SDSpolyacrylamide gel and subsequently transferred to nitrocellulose. Immunoblotting was accomplished using hybridoma supernatant containing mAbs L243 (23) or XD5.A11 (27). Binding of primary mAbs was detected using peroxidase-conjugated $\mathrm{F}\left(\mathrm{ab}^{\prime}\right)_{2}$ goat anti-mouse IgG + IgM (Jackson ImmunoResearch Laboratories) and a chemiluminescent substrate for peroxidase, ECL, (Amersham Corp., Arlington Heights, IL) followed by exposure to film.

\section{Results}

HLA-DR transfected BLS cells exhibit conformationally altered class II dimers. Defects in class II antigen structure have been linked with the impaired ability of both in vitro-generated (11, $13,14,28)$ and naturally occurring (BLS-1) (8) mutant cells to present peptides derived from exogenously supplied antigens. Previously we showed that BLS-1 cells expressing introduced DR4w4 and DR3 molecules failed to bind allele-specific antiDR3 and -DR4w4 mAbs that recognize conformation-dependent epitopes on these DR molecules (8). These conformation-dependent epitopes apparently reflect the specificity of selected antibodies to recognize intact peptide/class II complexes. Structurally altered peptide/class II complexes also show a marked reduction in ability to remain stable DR $\alpha \beta$ heterodimers after room temperature incubation in low concentrations of $\operatorname{SDS}(14,28)$. 


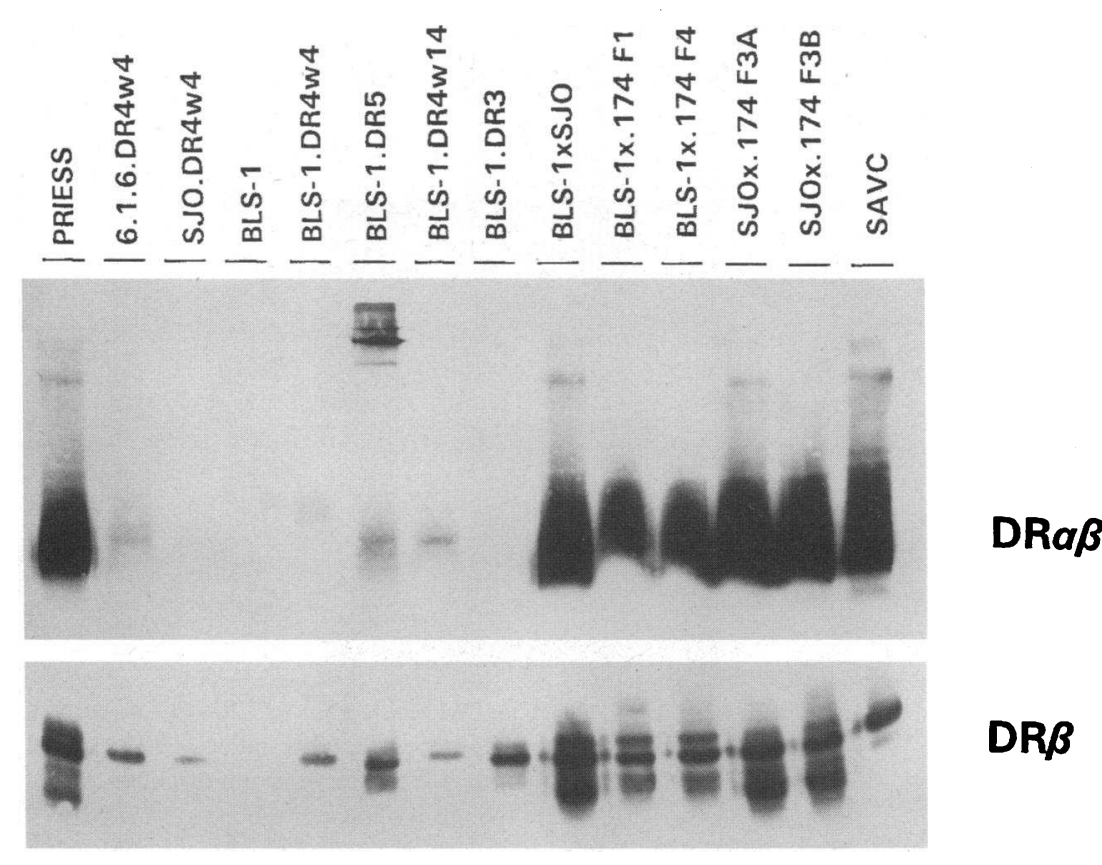

Figure 1. HLA-DR transfected BLS cells contain conformationally altered class II $\alpha \beta$ dimers that are unstable in SDS, and are rendered SDS-stable upon cell fusion. Top, detection of $60 \mathrm{kD}$ class II $\alpha \beta$ dimers in unboiled cell lysates in $0.2 \%$ SDS by immunoblotting with mAb L243. Bottom, detection of the $28 \mathrm{kD}$ DR $\beta$ chain in boiled and reduced cell lysates in $0.2 \%$ SDS by immunoblotting with $\mathrm{mAb}$ XD5.A11. Names of cell lines in each lane are depicted above both panels. Two independently fused BLS-1 $\times .174$ or SJO $\times .174$ hybrids are shown.

To investigate if abnormally conformed class II molecules are characteristic of different BLS complementation groups, genes encoding DR $\alpha$ (DRA1) and DR4w4 $\beta$ (DRB1*0401) were transferred into cells representative of two additional BLS complementation groups, 6.1.6 and SJO. We then investigated whether DR $\alpha \beta$ heterodimers with different allelic specificities, expressed in distinct BLS cells, would also exhibit this SDS instability. Aliquots of total cell lysate were incubated in either nonreducing SDS sample buffer at room temperature or boiled in reducing SDS sample buffer before SDS-PAGE and transfer to nitrocellulose. Immunoblotting was performed on the nonreduced samples using mAb L243 (Fig. 1, top) which recognizes mature DR heterodimers, and on the reduced samples using mAb XD5.A11 (Fig. 1, bottom), which recognizes DRB monomers.

The DR heterodimers in BLS-1, SJO, and 6.1.6 transferents, detectable by immunoblotting with mAb L243, showed greatly reduced stability in SDS as compared to the wild-type B-LCL Priess and Savc. No class II $\alpha \beta$ dimer was observed in BLS-1 cells transfected only with the DRA1 gene. Interestingly, although the amounts of DRB monomer were comparable in the different BLS- 1 transferents, the amount of SDS-stable DR heterodimer detectable varied significantly with the DR allele expressed. DR5 and DR4w14 dimers were more stable than DR4w4 dimers, while no DR3 dimers were detected.

The observation of residual stable DR4 and DR5 dimers in BLS cells suggests the binding of a subset of peptides even in these mutant APC. In contrast, stable DR3 dimers are not detected in BLS cells, a result also observed in several in vitro mutants defective in antigen presentation $(14,28)$. DR3 molecules in the mutant cells are preferentially loaded with a nested set of invariant chain-derived peptides (CLIP) that do not impart dimer stability in SDS $(29,30)$. Interestingly, consistent with our observation, HPLC analyses of peptides eluted from DR4w4 and DR5 molecules purified from the in vitro-generated mutant APC T2.DR4w4 and T2.DR5 revealed a minor proportion of peptides that were distinguishable from CLIP peptides and were not present in DR3 molecules purified from T2 cells (31). CLIP binds promiscuously to many class II alleles; how- ever, its avidity for DR4w4 and DR5 molecules is reduced compared with DR3 antigens (31). Thus, the presence of residual stable DR4 and DR5 dimers in BLS cells may reflect differential CLIP loading or retention by DR molecules in these cells as well as limited antigenic peptide binding.

BLS cells are deficient in HLA class II-mediated presentation of exogenously supplied native antigens. Since structurally altered class II molecules correlate with defects in the peptideclass II complex, the ability of DR4w4-transfected BLS cells to function in the class II-mediated presentation of two foreign protein antigens, TT and influenza virus hemagglutinin (HA), was then investigated (Fig. 2). Upon incubation with a preparation of proteolyzed TT (containing antigenic peptides), BLS1.DR4w4, 6.1.6.DR4w4, and SJO.DR4w4 cells were able to stimulate lymphokine production by a murine $\mathrm{T}$ cell hybridoma that is specific for TT in the context of DR4w4 (Fig. $2 A$ ). The ability of mutant BLS cells to present tetanus peptides was comparable to that of the wild-type DR4w4 B-LCL Priess; the presence of antigenic peptides in the proteolyzed tetanus was confirmed by incubation of the preparation with chemicallyfixed Priess cells (data not shown). In contrast, BLS-1.DR4w4, SJO.DR4w4, and 6.1.6.DR4w4 cells showed a marked reduction in ability to present peptide epitopes derived from native TT to this $\mathrm{T}$ cell hybridoma (Fig. $2 \mathrm{~A}$ ).

Similarly, after incubation with formalin-fixed influenza virus, the three DR-transfected BLS cells (BLS-1, SJO, and 6.1.6) were each deficient in presenting native $\mathrm{HA}$ to a $\mathrm{T}$ cell hybridoma recognizing DR4w4/HA307-319 complexes (Fig. $2 B$ ). The HA peptide 307-319 was presented with comparable efficiency by the mutant BLS and wild-type Priess cells. The small amount of $\mathrm{T}$ cell stimulation observed after incubation of 6.1.6.DR4w4 cells with native TT (Fig. $2 A$ ) was not observed after incubation of these cells with influenza virus, suggesting that the severity of the APC deficiency may be related to the nature of the protein antigen being processed and presented. Thus, cells representative of three distinct BLS complementation groups were deficient in the class II-mediated presentation of the native exogenous antigens, TT and influenza HA, although presentation of preprocessed antigenic peptides by these 

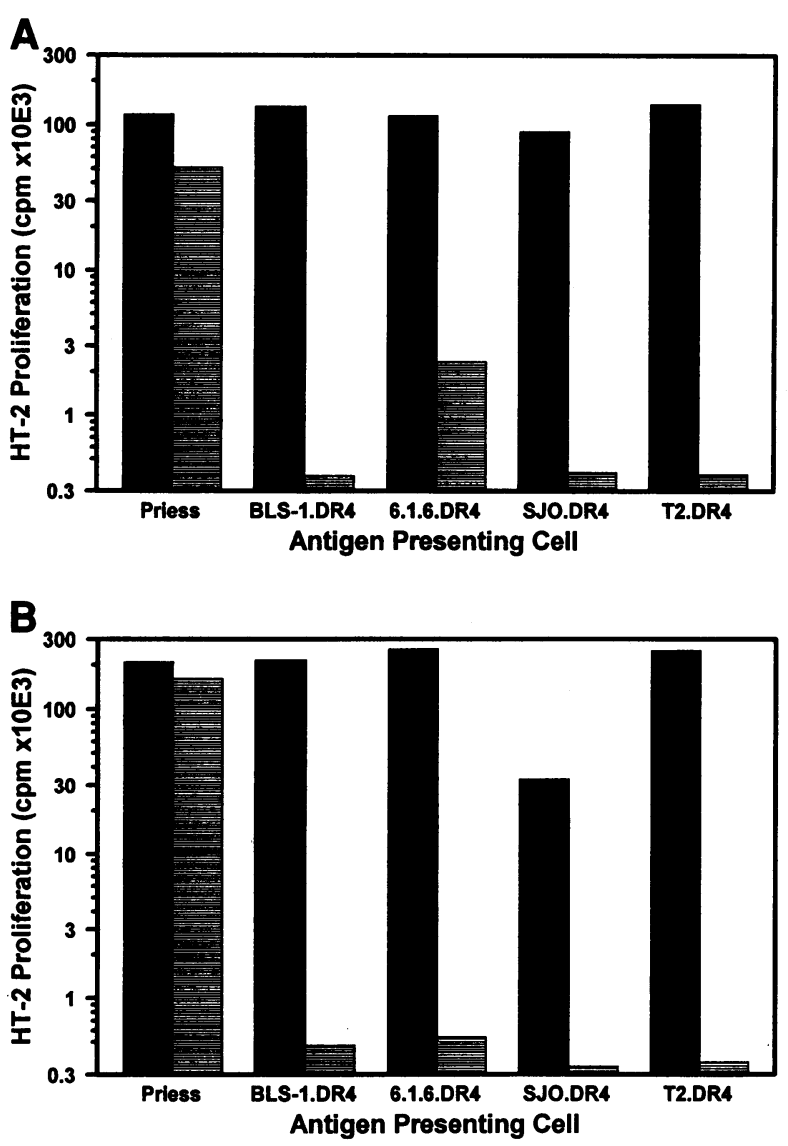

Figure 2. DR4w4-transfected BLS cells are unable to mediate class IIrestricted presentation of native exogenous protein antigens to $\mathrm{T}$ cells. $(A)$ Lymphokine production by a TT-specific T cell hybridoma in response to DR4w4-expressing APCs incubated with $0.4 \mu \mathrm{M}$ of a tryptic digest of TT peptides (solid bars) or native TT (hatched bars). (B) Lymphokine production by an influenza HA-specific $\mathrm{T}$ cell hybridoma in response to DR4w4-expressing APCs incubated with $1.0 \mu \mathrm{M} \mathrm{HA}$ 307-319 peptide (solid bars) or $50 \mu \mathrm{g} / \mathrm{ml}$ fixed influenza virus (hatched bars). Data are presented as proliferation of the IL-2/IL-4-dependent cell line, HT-2, in response to lymphokines produced by the T cell hybridomas. The data are mean values of $\left[{ }^{3} \mathrm{H}\right]$ thymidine incorporation from duplicate cultures in representative experiments; cpm values resulting from response of T cells to APCs in the absence of antigen have been subtracted.

cells was efficient. The inability of the mutant APC T2.DR4w4 cells to present either native TT or influenza HA to the DR4w4restricted T cells was comparable to the BLS cells (Fig. 2).

$B L S$ cells lack HLA-DM mRNA. The defects in class IImediated antigen presentation in BLS cells are similar to those observed in the in vitro-generated mutant cell lines 9.5 .3 (28), 2.2.93 (11), 721.174 (13), and T2 (14). These mutants each lack or have mutations in MHC-linked genes required for presentation of native class II-restricted antigens, recently identified as the DMA and DMB genes $(10,11)$. The DM genes map in the class II region of the MHC and contain 5' regulatory sequences that are similar to the promoter regions of the DR, $D Q$, and DP structural genes (12). Because the mutations in BLS cells are in trans-acting factors required for transcription of the class II structural genes, we hypothesized that BLS cells would also be deficient in expression of DM genes. Indeed, in a previous study, the SJO cell line from BLS group IV had

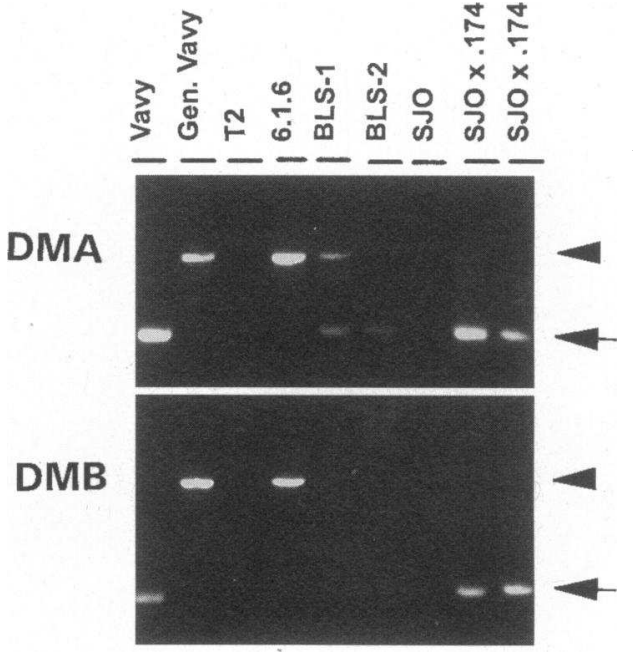

Figure 3. BLS cells lack HLA-DM RNA. (Top) PCR amplification of cDNA corresponding to the DMA gene; the arrow indicates the expected DMA product of $340 \mathrm{bp}$. (Bottom) PCR amplification of cDNA corresponding to the DMB gene; the arrow indicates the expected DMB product of $320 \mathrm{bp}$. Names of cell lines in each lane are indicated above both panels. Two independently fused SJO $\times .174$ hybrids were analyzed. The higher molecular weight band present in some samples (indicated by the arrowheads) is likely to have been amplified from genomic DNA contaminating the cDNA preparations since bands of the same size are amplified directly from genomic DNA (lane marked "Gen. Vavy").

been examined for DM mRNA, with evidence suggesting these cells are unable to produce both DMA and DMB (32).

A PCR assay was used to detect DMA and DMB mRNA in cells representative of the four BLS complementation groups, BLS-1, BLS-2, 6.1.6, and SJO (Fig. 3). The amount of PCRamplified signal corresponding to the expected DMA and DMB products in each BLS cell was compared to that of a wild-type $B$ cell line Vavy and the DM-deletion mutant T2. All four BLS cells were deficient in DMB mRNA (Fig. 3, bottom). In contrast, assays of BLS-1, BLS-2, and 6.1.6 showed a weak band corresponding to DMA mRNA, while SJO lacked this band (Fig. 3, top). The identity of the putative DMA band in BLS-1 cells was confirmed by DNA sequencing of the PCR product. The difference in intensity of DMA bands between wild-type B cells and these BLS cells suggests that BLS cells contain reduced amounts of steady-state DMA mRNA. This observation is consistent with the report that BLS-1 cells also contain reduced but detectable amounts of DQA1 mRNA (17). These data indicate that the trans-acting factors missing in each of the four BLS cells, representing all four BLS complementation groups, are required for transcriptional activation of both the DMA and DMB genes, although DMA expression is apparently regulated by additional parameters.

Fusion of BLS cells with the HLA class II deletion mutant 721.174 restores a wild-type APC phenotype. Cell fusion studies have been used to demonstrate that the defects in class II gene transcription in BLS cells are linked to the absence of transacting factors encoded outside of the $\operatorname{MHC}(6,7)$. To investigate if a functional APC phenotype could also be restored by trans-acting factors, we fused BLS-1 (DR3,5) or SJO (DR5,7) cells with 721.174. The latter line contains a deletion in the class II region of the MHC that removes both structural class II genes and the genes (HLA-DMA and -DMB) required for 
Table I. Fusion of BLS Cells with Mutant .174 Restores Expression of DR Epitopes

\begin{tabular}{lcc}
\hline & \multicolumn{2}{c}{$\begin{array}{c}\text { mAb recognition of DR epitopes } \\
\text { (mean channel fluorescence) }\end{array}$} \\
\cline { 2 - 3 } \multicolumn{1}{c}{ Cell line } & All DR (L243) & DR3 (16.23) \\
\hline 174 & 8 & 10 \\
BLS-1 & 12 & 15 \\
BLS-1.DR3 & 680 & 84 \\
BLS-1 $\times .174$ F1 & 818 & 460 \\
Mat & 2996 & 734 \\
SJO.DR4 $\beta$ & 13 & nd \\
SJO $\times .174$ F3A & 1558 & nd \\
\end{tabular}

Anti-DR mAb binding to live cells was detected by flow cytometry. Mat is a wild-type DR3 B-LCL. BLS-1 contains an endogenous DR3 gene that is expressed upon fusion with .174 . SJO.DR4 $\beta$ contains only a DRB $1 * 0401$ transgene. $n d$, not determined.

presentation of exogenous protein antigens, but is presumably intact for the trans-acting regulatory genes defective in the BLS lines. Fused hybrid cells BLS- $1 \times .174$ and SJO $\times .174$ were isolated and assessed for expression of endogenous class II molecules in the BLS cells. The hybrid cells expressed abundant amounts of endogenous DR molecules detectable with mAb L243 that recognizes mature DR heterodimers (Table I) and by mAb SFR3-DR5 (33) that recognizes DR5 molecules (data not shown). The SJO cells also contained a transgene encoding DR4w $4 \beta$ chains; however, surface expression of DR4w4 molecules was only observed after transcriptional activation of endogenous DR alpha chain expression in the hybrid cells (Table I and data not shown).

The DR $\alpha \beta$ structure in these hybrid cells, as well as the previously characterized BLS $1 \times$ SJO hybrid cells $(8)$, was investigated using the assay for class II dimer stability described above. Upon fusion of BLS-1 or SJO cells with each other or with 721.174, the amount of SDS-stable dimer corresponding to the endogenous DR gene products was present in wild-type amounts (Fig. 1, top). The structure of endogenously expressed DR3 molecules in the BLS-1 1.174 fused cells was also investigated using the conformationally-sensitive anti-DR3 mAb 16.23. Fusion of BLS-1 with .174 resulted in production of DR3 molecules containing the mAb 16.23 epitope; the amount of mAb 16.23 bound was significantly greater than that bound to BLS-1.DR3 transferents and comparable to the wild-type DR3 B cell line Mat (Table I). Identical results were previously obtained after fusion of BLS-1 and SJO (8). Taken together, these data indicate that fusion with 721.174 results in the production of DR molecules that have the same conserved structural features as DR molecules present in wild-type B cells, a phenotype that is markedly distinct from DR molecules in BLS and mutant APC such as 721.174. These results are simply explained if the trans-acting factors that mediate class II structural gene transcription also mediate transcription of genes required for a functional APC phenotype.

Presentation of exogenous native antigen was also restored in SJO $\times .174$ hybrid cells (Fig. 4). In marked contrast to SJO.DR4w4 cells, the SJO $\times .174$ cells were able to present native TT (Fig. $4 A$ ) or native HA (Fig. $4 B$ ) to the appropriate DR4w4-restricted murine T cell hybridomas. While the presentation of HA peptides, either preprocessed or derived after cellu-
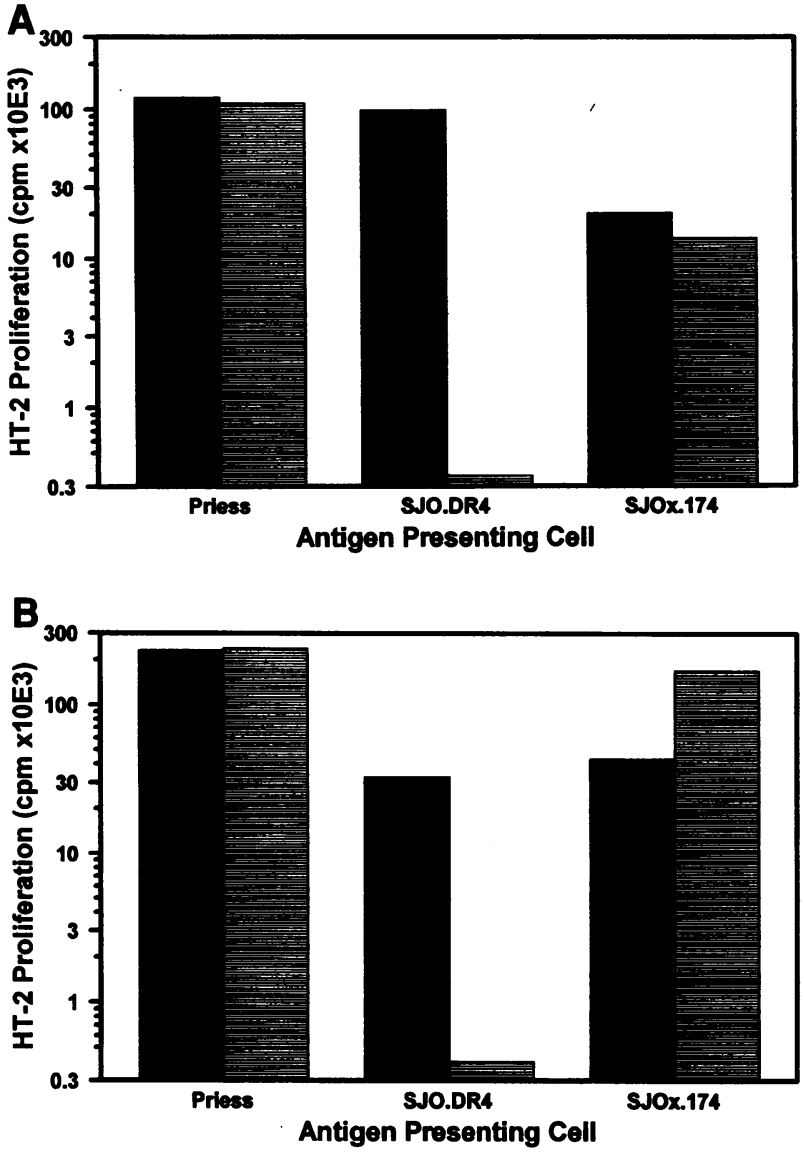

Figure 4. Fusion of SJO cells with the HLA class II deletion mutant 721.174 restores the ability of SJO to present native exogenous antigens to T cells. $(A)$ Lymphokine production by a TT-specific T cell hybridoma in response to DR4w4 B cell line Priess, SJO.DR4w4 or hybrid SJO $\times .174$ cells incubated with $0.4 \mu \mathrm{M}$ TT peptides (solid bars) or $0.8 \mu \mathrm{M}$ native TT (hatched bars). (B) Lymphokine production by an influenza HA-specific T cell hybridoma in response to Priess,

SJO.DR4w4 or SJO $\times .174$ cells incubated with $1.0 \mu \mathrm{M}$ HA 307-319 (solid bars) or $50 \mu \mathrm{g} / \mathrm{ml}$ fixed influenza virus (hatched bars). Data are presented as proliferation of the IL-2/IL-4-dependent cell line, HT-2. Data are mean values of $\left[{ }^{3} \mathrm{H}\right]$ thymidine incorporation from duplicate cultures in representative experiments.

lar processing of influenza virus, was comparable in Priess and SJO $\times .174$ cells, presentation of TT (native or trypsin-digested) was reduced in the SJO $\times .174$ cells, relative to wildtype B cells, in multiple experiments.

The nearly wild-type APC phenotype of the hybrid cells prompted us to check the hybrid cells for the amount of expression of HLA-DMA and -DMB, the MHC-linked genes required for functional antigen processing. Fusion of SJO with 721.174 resulted in the presence of PCR-amplified products corresponding to the DMA and DMB genes (Fig. 3). Since 721.174 has a homozygous deletion of the MHC class II region that includes the DMA and DMB loci, these data are consistent with the explanation that the trans-acting factor present in 721.174 that restores class II gene transcription in SJO also restores transcription of the DMA and DMB genes.

\section{Discussion}

An HLA class II null phenotype typifies each of the type II BLS complementation groups, yet at least four distinct genetic 
defects manifest as this immunodeficiency. Restoration of class II expression in BLS lines by transfection of fully competent exogenous class II genes restores the ability of these cells to present antigenic peptides to $\mathrm{T}$ lymphocytes. Notably, however, class II-transfected BLS cells remain incompetent to present protein antigens to the same $\mathrm{T}$ lymphocytes, indicating a persistent deficit in antigen processing and presentation. We have evaluated B cell lines from different BLS complementation groups to characterize this antigen presentation phenotype; and found that all transfected BLS cells tested present preprocessed peptides to $\mathrm{T}$ lymphocytes, but do not process and present epitopes derived from whole proteins. Genetic complementation of the BLS defect by fusion with a cell lacking genes in the class II region of the MHC restored both class II expression and a fully competent APC phenotype. Thus, each of the molecular defects that silences class II structural gene expression in BLS also results in a defective APC phenotype, providing strong evidence for coregulation of these pathways.

The defective APC phenotype in BLS has a structural correlate, as demonstrated by analyses of class II $\alpha \beta$ dimer stability and conformation-dependent anti-DR3 mAb epitopes. After room temperature incubation in low concentrations of detergent, DR molecules in all BLS transferent lines tested showed greatly reduced stability as compared to class II molecules in wild-type B cells; fusion of the DR-transfected BLS cells with other class II negative mutants again restored a wild-type phenotype, in this case the stability of the class II $\alpha \beta$ dimer. Recent studies of DM-deficient mutant T2 and 9.5.3 cells expressing the murine class II molecules, I-A ${ }^{d}$ or I-A ${ }^{k}$, have shown that I-A $\alpha \beta$ dimer stability in SDS may not be strictly correlated with the ability to mediate presentation of native exogenous antigens $(34,35)$. I-A ${ }^{d}$ molecules, but not $\mathbf{I}-A^{k}$ molecules $(34,35)$, exhibited marked SDS-instability yet retained the capacity to present certain epitopes derived from exogenous protein antigens. Conversely, SDS-stable $I-A^{k}$ molecules were unable to mediate presentation of other epitopes (35). This differential behavior of the murine I-A molecules in the mutant human cell lines may be due to differences in avidity of these I-A alleles for the invariant chain-derived CLIP peptides. Although all DR molecules showed greatly reduced SDS-stability in the BLS-1 cell line, we observed subtle allelic differences in DR $\alpha \beta$ stability that may correlate with the differential avidity of these DR alleles for the CLIP peptides (31) and, therefore, may reflect allele-specific formation of selected stable peptide/class II complexes. However, in deficient APC containing human class II alleles, SDS-unstable class II $\alpha \beta$ dimers are universally observed.

The genetic defects in three of the BLS complementation groups are known; each involves defective transcription factors required for coordinate expression of HLA class II genes. The transcription factor defective in cells of complementation groups I and IV, such as BLS-1 and SJO, is apparently RFX, since purified RFX protein complements DRA transcription in these cells (36). An RFX heterodimer most likely interacts with consensus $\mathrm{X}$ box elements present in HLA class II upstream regulatory regions (36). A deficiency in an $X$ box binding protein in the 6.1 .6 cell line, complementation group III, has also been reported (37). The defective APC phenotype manifested in each of these three distinct complementation groups suggests that genes required for antigen presentation are coregulated by the same set of transactivators as the class II structural genes. Cells in BLS complementation group II, such as BLS2 , are defective in the CIITA protein (38), a protein that may not directly bind DNA (38) but which likely acts in both constitutive and $\gamma$-interferon-stimulated class II transcriptional activation $(39,40)$. Since BLS- 2 cells lack DMB mRNA, CIITA is likely to be involved in regulation of genes required for antigen presentation ( see below), suggesting that these cells would display a defective APC phenotype upon transfection of class II genes. Indeed, a recent report (41) shows that transfection of the CIITA gene into BLS-2 cells results in endogenous DR and DM gene expression, indicating coordinate transcriptional regulation of these diverse class II genes.

Since the defective APC phenotype in BLS cells is functionally similar to the phenotype of previously described in vitrogenerated cell mutants defective in the HLA-DMA and -DMB genes, we evaluated mRNA levels for DMA and DMB in each of the four BLS complementation groups. mRNA for DMB was indeed absent in all BLS cells tested, and was restored by transacting factors upon fusion with a DM-deficient cell line, coordinate with class II expression and APC function. Amounts of DMA mRNA, in contrast, were low but variable in the distinct BLS cell lines tested and also apparently increased in the SJO $\times .174$ hybrid cells. This discrepancy between HLA class II A and $B$ gene regulation may be a general phenomenon, since expression of both the DQA1 (17) and DQA2 (42) genes have also been previously described in cells lacking expression of DQB. Thus, these data suggest that the absence of functional HLA-DM proteins in the BLS cells may account for the defective APC phenotype.

Interestingly, the upstream DNA sequence proximal to the first exon of the DMA and DMB genes contains HLA class IIlike consensus sequence elements, the $\mathrm{X}$ and $\mathrm{Y}$ boxes, shared with class II DR, DQ, and DP genes (12); and like the class II structural and invariant chain genes, the DM genes are likely to be coordinately upregulated in response to $\gamma$-IFN (43). The presence of these similar regulatory elements supports the interpretation that each of the transcription factors defective in the BLS complementation groups also act on the promoter elements for the DMA and DMB genes, and in this way, function as a coordinate regulator of the separate pathways of structural class II gene expression and functional antigen processing and presentation. However, the transcription factors absent in the BLS cells may be involved in regulation of additional genes required for optimal antigen processing and presentation. Accordingly, we have observed a decrease in amounts of intracellular invariant chain in each of the four BLS cells described here (S. Kovats and J. S. Blum, unpublished observations), consistent with coregulation of invariant chain gene transcription via $5^{\prime}$ $\mathrm{X}$ and $\mathrm{Y}$ box consensus elements (16).

The conservation of shared transcriptional regulatory elements provides a simple and effective mechanism for coregulation of these two distinct pathways, and is thus an interesting genetic model for the coevolution of distinct genes whose products participate in different aspects of a common intracellular process. As the class II $\alpha \beta$ dimer stability experiments demonstrate, products of genes involved in APC function appear to directly interface with the products of the class II structural genes themselves to produce fully competent APC. Furthermore, both the structural class II pathway and the antigen processing/presentation pathway likely contribute to the clinical immunodeficiency associated with BLS, implying that therapies directed at the coordinate regulatory elements rather than the class II genes themselves are needed to fully correct the functional deficits. 


\section{Acknowledgments}

We thank P. Whiteley and D. Zaller for T cell hybridomas, R. DeMars and R. Greenwood for 721.174 cells, and J. Lee for BLS-1 and BLS-2 cells. We also thank our colleagues for helpful discussions and $\mathrm{H}$. Chase for manuscript preparation.

This study was supported by National Institutes of Health grants (AI-33418 and AI-31241) and the Arthritis Foundation (J. S. Blum). S. Kovats was supported by a National Research Service Award from the National Institutes of Health.

\section{References}

1. Lisowska-Grospierre, B., D. J. Charron, C. de Preval, A. Durandy, C. Griscelli, and B. Mach. 1985. A defect in the regulation of major histocompatibility complex class II gene expression in human HLA-DR negative lymphocytes from patients with combined immunodeficiency syndrome. J. Clin. Invest. 76:381-385.

2. de Preval, C., B. Lisowska-Grospierre, M. Loche, C. Griscelli, and B. Mach. 1985. A trans-acting class II regulatory gene unlinked to the MHC controls expression of HLA class II genes. Nature (Lond.) 318:291-293.

3. Klein, C., B. Lisowska-Grospierre, F. LeDeist, A. Fischer, and C. Griscelli. 1993. Major histocompatibility complex class II deficiency: clinical manifestations, immunologic features, and outcome. J. Pediatr. 123:921-928.

4. Hume, C. R., and J. S. Lee. 1989. Congenital immunodeficiencies associated with absence of HLA class II antigens on lymphocytes result from distinct mutations in trans-acting factors. Human Immunol. 26:288-309.

5. Yang, Z., R. S. Accolla, D. Pious, B. J. Zegers, and J. L. Strominger. 1988. Two distinct loci regulating class II gene expression are defective in human mutant and patient cell lines. EMBO (Eur. Mol. Biol. Organ.) J. 7:1965-1972.

6. Benichou, B., and J. L. Strominger. 1991. Class II-antigen-negative patien and mutant B-cell lines represent at least three, and probably four, distinct genetic defects defined by complementation analysis. Proc. Natl. Acad. Sci. USA 88:4285-4288.

7. Seidl, C., C. Saraiya, Z. Osterweil, Y. P. Fu, and J. S. Lee. 1992. Genetic complexity of regulatory mutants defective for HLA class II gene expression. $J$. Immunol. 148:1576-1584.

8. Kovats, S., S. Drover, W. Marshall, D. Freed, P. Whiteley, G. T. Nepom and J. S. Blum. 1994. Coordinate defects in HLA class II expression and antigen presentation in bare lymphocyte syndrome. J. Exp. Med. 179:2017-2022.

9. Cresswell, P. 1994. Assembly, transport, and function of MHC class II molecules. Annu. Rev. Immunol. 12:259-293.

10. Morris, P., J. Shaman, M. Attaya, M. Amaya, S. Goodman, C. Bergman, J. J. Monaco, and E. Mellins. 1994. An essential role for HLA-DM in antigen presentation by class II major histocompatibility molecules. Nature (Lond.). 368:551-554.

11. Fling, S. P., B. Arp, and D. Pious. 1994. HLA-DMA and -DMB genes are both required for MHC class II/peptide complex formation in antigen-presenting cells. Nature (Lond.). 368:554-558.

12. Kelly, A. P., J. J. Monaco, S. G. Cho, and J. Trowsdale. 1991. A new human HLA class II-related locus, DM. Nature (Lond.). 353:571-573.

13. Ceman, S., R. Rudersdorf, E. O. Long, and R. DeMars. 1992. MHC class II deletion mutant expresses normal levels of transgene encoded class II molecules that have abnormal conformation and impaired antigen presentation ability. $J$. Immunol. 149:754-761.

14. Riberdy, J. M., and P. Cresswell. 1992. The antigen-processing mutant T2 suggests a role for MHC-linked genes in class II antigen presentation. $J$. Immunol. 148:2586-2590.

15. Mellins, E., S. Kempin, L. Smith, T. Monji, and D. Pious. 1991. A gene required for class II restricted antigen presentation maps to the major histocompatibility complex. J. Exp. Med. 174:1607-1615.

16. Doyle, C., P. J. Ford, P. D. Ponath, T. Spies, and J. L. Strominger. 1990. Regulation of the class II-associated invariant chain gene in normal and mutant B lymphocytes. Proc. Natl. Acad. Sci. USA. 87:4590-4594.

17. Hume, C. R., L. A. Shookster, N. Collins, R. O'Reilly, and J. S. Lee. 1989. Bare lymphocyte syndrome: altered HLA class II expression in B cell lines derived from two patients. Human Immunol. 25:1-11.

18. Bull, M., A. vanHoef, and J. Gorski. 1990. Transcription analysis of class II human leukocyte antigen genes from normal and immunodeficient B lymphocytes, using polymerase chain reaction. Mol. Cell. Biol. 10:3792-3796.

19. Gladstone, P. and D. Pious. 1980. Identification of a trans-acting function regulating HLA-DR expression in a DR-negative B cell variant. Somatic Cell Genetics. 6:285-298.
20. Salter, R. D., D. N. Howell, and P. Cresswell. 1985. Genes regulating HLA class I antigen expression in T-B lymphoblast hybrids. Immunogenet. 21:235-246.

21. Kwok, W. W., D. Schwarz, B. S. Nepom, P. S. Thurtle, R. A. Hock, and G. T. Nepom. 1988. HLA-DQ molecules form $\alpha-\beta$ heterodimers of mixed allotype. J. Immunol. 141:3123-3127.

22. DeMars, R., C. C. Chang, S. Shaw, P. J. Reitnauer, and P. M. Sondel. 1984. Homozygous deletions that simultaneously eliminate expressions of class I and class II antigens of EBV-transformed B-lymphoblastoid cells. I. Reduced proliferative responses of autologous and allogeneic $\mathrm{T}$ cells to mutant cells that have decreased expression of class II antigens. Hum. Immunol. 11:77-97.

23. Lampson, L. A., and R. Levy. 1980. Two populations of la-like molecules on a human B cell line. J. Immunol. 135:293-299.

24. Johnson, J. P., T. Meo, G. Riethmuller, D. J. Schendel, and R. Wank 1982. Direct demonstration of an HLA-DR allotypic determinant on the low molecular weight (beta) subunit using a mouse monoclonal antibody specific for DR3. J. Exp. Med. 156:104-111.

25. Woods, A., H. Y. Chen, M. E. Trumbauer, A. Sirotina, R. Cummings, and D. M. Zaller. 1994. Human major histocompatibility complex class II-restricted T cell responses in transgenic mice. J. Exp. Med. 180:173-181.

26. Kappler, J., B. Skidmore, J. White, and P. Marrack. 1981. Antigen-inducible, H-2-restricted, interleukin 2-producing $\mathrm{T}$ cell hybridomas. Lack of independent antigen and H-2 recognition. J. Exp. Med. 153:1198-1214.

27. Radka, S. F., C. E. Machamer, and P. Cresswell. 1984. Analysis of monoclonal antibodies reactive with human class II beta chains by two-dimensional electrophoresis and Western blotting. Hum. Immunol. 10:177-186.

28. Mellins, E., L. Smith, B. Arp, T. Cotner, E. Celis, and D. Pious. 1990. Defective processing and presentation of exogenous antigens in mutants with normal HLA class II genes. Nature (Lond.). 343:71-74.

29. Riberdy, J. M., J. R. Newcomb, M. J. Surman, J. A. Barbosa, and P. Cresswell. 1992. HLA-DR molecules from an antigen-processing mutant cell line are associated with invariant chain peptides. Nature (Lond.). 360:474-477.

30. Sette, A., S. Ceman, R. T. Kubo, K. Sakaguchi, E. Appella, D. F. Hunt, T. A. Davis, H. Michel, J. Shabanowitz, R. Rudersdorf, H. M. Grey, and R. DeMars. 1992. Invariant chain peptides in most HLA-DR molecules of an antigenprocessing mutant. Science (Wash. DC) 258:1801-1804.

31. Avva, R. R. and P. Cresswell. 1994. In vivo and in vitro formation and dissociation of HLA-DR complexes with invariant chain-derived peptides. Immunity. 1:763-774.

32. Nocera, A., S. Barocci, R. De Palma, and J. Gorski. 1993. Analysis of transcripts of genes located within the HLA-D region in B cells from an HLAsevere combined immunodeficiency individual. Human Immunol. 38:231-234.

33. Radka, S. F., C. Machamer, P. Cresswell, D. D. Kostyu, F. E. Ward, and D. B. Amos. 1983. SFR3-DR5, a monoclonal antibody with HLA-DR5 specificity. J. Immunol. 130:1863-1866.

34. Stebbins, C. C., G. E. Loss, Jr., C. G. Elias, A. Chervonsky, and A. J. Sant. 1995. The requirement for DM in class II-restricted antigen presentation and SDS-stable dimer formation is allele and species dependent. J. Exp. Med. 181:223-234.

35. Brooks, A. G., P. L. Campbell, P. Reynolds, A. M. Gautam, and J. McCluskey. 1994. Antigen presentation and assembly by mouse I-A ${ }^{k}$ class II molecules in human APC containing deleted or mutated HLA DM genes. J. Immunol. 153:5382-5392.

36. Durand, B., M. Kobr, W. Reith, and B. Mach. 1994. Functional complementation of major histocompability complex class II regulatory mutants by the purified X-box-binding protein RFX. Mol. Cell. Biol. 14:6839-6847.

37. Stimac, E., S. Urieli-Shoval, S. Kempin, and D. Pious. 1991. Defective HLA DRA $X$ box binding in the class II transactive transcription factor mutant 6.1.6 and in cell lines from class II immunodeficient patients. J. Immunol. 146:4398-4405

38. Steimle, V., L. A. Otten, M. Zufferey, and B. Mach. 1993. Complementation cloning of an MHC class II transactivator mutated in hereditary MHC class II deficiency (or bare lymphocyte syndrome). Cell. 75:135-146.

39. Steimle, V., C.-A. Siegrist, A. Mottet, B. Lisowska-Grospierre, and B. Mach. 1994. Regulation of MHC class II expression by interferon-gamma mediated by the transactivator gene CIITA. Science (Wash. DC). 265:106-109.

40. Chin, K.-C., C. Mao, C. Skinner, J. L. Riley, K. L. Wright, C. S. Moreno, G. R. Stark, J. M. Boss, and J. P.-Y. Ting. 1994. Molecular analysis of G1B and G3A IFN gamma mutants reveals that defects in CIITA or RFX result in defective class II MHC and li gene induction. Immunity. 1:687-697.

41. Chang, C.-H., and R. A. Flavell. 1995. Class II transactivator regulates the expression of multiple genes involved in antigen presentation. J. Exp. Med. 181:765-767.

42. Yu, L., and M. J. Sheehy. 1991. The cryptic HLA-DQA2 ("DXalpha") gene is expressed in human B cell lines. J. Immunol. 147:4393-4397.

43. Mao, C., D. Davies, I. M. Kerr, and G. R. Stark. 1993. Mutant human cells defective in induction of major histocompatibility complex class II genes by interferon gamma. Proc. Natl. Acad. Sci. USA. 90:2880-2884. 